\title{
THE APPARENT AND PARTIAL MOLAR VOLUMES OF SODIUM CARBOXYMETHYLCELLULOSE IN ACETONITRILE-WATER MIXED SOLVENT MEDIA
}

\author{
P. Nandi and C. Das ${ }^{*}$ \\ Department of Chemistry, NBBD College, Tadong, Gangtok- 737102, Sikkim, India \\ *E-mail: cdaschem@gmail.com
}

\begin{abstract}
The volumetric properties of Sodium Carboxymethylcellulose (NaCMC) in acetonitrile-water mixed solvent media containing $(0.10,0.20$, and 0.40$)$ volume fractions of acetonitrile were studied by measuring the density of solutions at temperatures $(298.15,308.15,313.15$, and 318.15$) \mathrm{K}$. Besides studying the solvation behavior of the counterions, the study was focused on investigating the interactions between the counterion and the polyion. In the specified temperature range, a linear increase for apparent molar volume $\left(\phi_{\mathrm{v}}\right)$ with increasing polyelectrolyte concentration was observed in a medium with fixed volume fractions of acetonitrile. However, at a given temperature as the medium became richer in acetonitrile the limiting partial molar volumes $\left(\bar{v}_{2}^{0}\right)$ registered a decrease. But, when a polyelectrolyte solution in a fixed medium underwent a step-wise increase in temperature then $\bar{V}_{2}^{-0}$ values increased thereby leading to the inference that a temperature-induced desolvation of the counterions results in more counterion binding at higher temperatures.

Keywords: Sodium Carboxymethylcellulose, Mixed Solvent Media, Partial Molar Volume, Counterion Binding, Ion-Solvent Interactions
\end{abstract}

(C) RASĀYAN. All rights reserved

\section{INTRODUCTION}

Polyelectrolytes have numerous techno-industrial usages and they motor the molecules which are necessary for the survival of living organisms. ${ }^{1-5}$ As such they have attracted the attention of many researchers. However, the properties of polyelectrolyte solutions deviate considerably from those of neutral polymeric solutions and those of simple electrolytic solutions. And the chief factors responsible for determining the properties of polyelectrolyte solutions are (i) the electro-repulsive forces that operate in between the ions of a polymer chain and (ii) the two-ways exchanges that take place in between the polyions and counterions. Of these, the first factor causes the polymer chain to expand. But the second factor not only operates in the binding of counterion, but it also has its role in determining the degree of solvation besides influencing the structure of the solvent lying near the polyelectrolytes. Finally, each of these factors in isolation affects the relative permittivity of the solvent media. When a number of mixed solvents are taken then one can observe systematic variation in the relative permittivity of the media. Using this variation one can investigate the interactions that go on in the polyelectrolyte solutions. ${ }^{6-9}$

Since the thermodynamic properties of polyelectrolyte solutions are useful in illuminating the ongoing structural interactions in solutions, they have been made use in the present investigation. Examples would make the matter clear. If the study of partial molar volumes ${ }^{10-14}$ of solutes under infinite dilution facilitates the understanding of solute-solvent and solvent-solvent interactions, then the solute-solute interactions can be studied using concentration dependence of apparent molar volume ${ }^{14-17}$ of solute. The present investigation is geared towards the understanding of polyion-counterion interactions as well as the solvation behavior of the counterions using the apparent and partial molar volumes of Sodium Carboxymethylcellulose ( $\mathrm{NaCMC}$ ) in acetonitrile-water mixtures. To do so, the solution densities of

Rasayan J. Chem., 12(4), 2039-2046(2019)

http://dx.doi.org/10.31788/RJC.2019.1245388

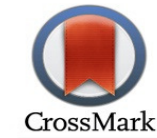


$\mathrm{NaCMC}$ were measured and the apparent molar volumes of polyelectrolyte solutions were calculated at experimental temperatures.

\section{EXPERIMENTAL}

Acetonitrile (purchased from E. Merck, India with a purity $>99 \%$ ) was fractionally distilled with phosphorous pentoxide. The middle fraction was collected and redistilled with calcium hydride. The density and viscosity co-efficient of the distilled acetonitrile at $308.15 \mathrm{~K}$ were $0.76570 \pm 0.00003{\mathrm{~g} . \mathrm{cm}^{-3}}^{-3}$ and $0.31260 \pm 0.00004 \mathrm{mPa}$.s respectively, values which conformed with the physical properties of the distilled acetonitrile as reported by the literature. ${ }^{18}$ The mixed solvents were prepared with triple-distilled water with specific conductance value $\sim 10^{-6} \mathrm{~S} . \mathrm{cm}^{-1} 1$. The measured values of density and viscosity of the mixed solvents at $(298.15,308.15,313.15$, and 318.15) $\mathrm{K}$ are shown in Table-1. With the help of density and relative permittivity values of the pure solvents ${ }^{18,19}$ at experimental temperatures and following the method of literature, ${ }^{20}$ the relative permittivities of the solvent mixtures were evaluated and are also incorporated in Table-1.

Table-1: Physical Properties of Acetonitrile-Water Mixtures Containing (0.10, 0.20, And 0.40) Volume Fraction of Acetonitrile at $(298.15,308.15,313.15$, And 318.15) K

${ }^{a}$ Evaluated following the method as represented in ref 20.

\begin{tabular}{|c|c|c|c|}
\hline$T / \mathrm{K}$ & $\rho_{0} /{\mathrm{g} . \mathrm{cm}^{-3}}^{-3}$ & $\eta_{0} / \mathrm{mPa} . \mathrm{s}$ & $D^{a}$ \\
\hline \multicolumn{4}{|c|}{$\phi_{1}=0.10$} \\
\hline 298.15 & 0.98307 & 1.0642 & 75.62 \\
\hline 308.15 & 0.98096 & 0.8766 & 72.15 \\
\hline 313.15 & 0.97893 & 0.7622 & 69.89 \\
\hline 318.15 & 0.97625 & 0.6733 & 68.27 \\
\hline \multicolumn{4}{|c|}{$\phi_{1}=0.20$} \\
\hline 298.15 & 0.97046 & 1.1003 & 72.19 \\
\hline 308.15 & 0.96485 & 0.9067 & 67.94 \\
\hline 313.15 & 0.96200 & 0.7833 & 66.38 \\
\hline 318.15 & 0.95912 & 0.6984 & 64.84 \\
\hline \multicolumn{4}{|c|}{$\phi_{1}=0.40$} \\
\hline 298.15 & 0.93800 & 1.0398 & 63.48 \\
\hline 308.15 & 0.92362 & 0.8200 & 60.07 \\
\hline 313.15 & 0.91730 & 0.6803 & 58.87 \\
\hline 318.15 & 0.91542 & 0.5611 & 57.32 \\
\hline
\end{tabular}

$\mathrm{NaCMC}$ (purchased from Sigma-Aldrich, USA) had an average molecular weight $\left(M_{\mathrm{w}}\right) \sim 90,000$ and 0.70 carboxymethyl groups per anhydroglucose unit. Fresh stocks solutions were used for each measurement in order to avoid problems associated with aging and contamination of the solutions from a microorganism, since such observations do occur in the dilute polyelectrolyte solutions. ${ }^{21}$

For all density measurements, an Ostwald-Sprengel type pycknometer (bulb volume of $25 \mathrm{~cm}^{3}$ and capillary diameter $1 \mathrm{~mm}$ ) was used. The pycknometer was standardized at the experimental temperatures with distilled water, methanol, and acetonitrile. Densities of the solutions were measured in a thermostatic water bath having an accuracy of $\pm 0.005 \mathrm{~K}$. The precision of the density measurements was always within $\pm 3 \times 10^{-5} \mathrm{~g} \cdot \mathrm{cm}^{-3}$.

\section{RESULTS AND DISCUSSION}

Thermodynamic quantities like partial or apparent molar volumes of the solute ${ }^{10}$ are often used to study the structural interactions of polyions in solutions. In general, the partial molar volume $\left(\bar{V}_{2}^{-}\right)$of the solute in a solution is the change in volume per mole of solute added to the mixture at constant temperature, 
RASĀYAN J. Chem.

Vol. 12 | No. 4 |2039 - 2046| October - December | 2019

pressure, and composition, i.e., $\bar{V}_{2}^{0}=\left(\partial V / \partial n_{2}\right)_{T, P, n_{1}}$. On the other hand, the formal contribution of the solute to the total volume of the solute-solvent system is defined as the apparent molar volumes $\left(\phi_{\mathrm{v}}\right)$. The apparent molar volume of the polyelectrolyte in solution is amenable to calculation using the following equation:

$$
\phi_{\mathrm{v}}=\frac{M}{\rho_{0}}-1000 \frac{\left(\rho-\rho_{0}\right)}{c_{\mathrm{p}} \rho_{0}}
$$

Where, $c_{\mathrm{p}}$ is the polyelectrolyte concentration (in monomolarity), $M$ the molar mass of the repeating unit of NaCMC with 0.70 carboxymethyl groups per anhydroglucose unit, $\rho$ and $\rho_{0}$ are the density of the solution and solvent respectively. Table- 2 shows the apparent molar volume of the NaCMC in different acetonitrile-water mixed solvents at various temperatures.

Table-2: Concentration, Density, and Apparent Molar Volume of NaCMC in Acetonitrile-Water Mixtures Containing (0.10, 0.20, And 0.40) Volume Fraction of Acetonitrile at $(298.15,308.15,313.15$, And 318.15) K

\begin{tabular}{|c|c|c|c|}
\hline$T / \mathrm{K}$ & $c /$ monomol. $^{-1}$ & $\rho / \mathrm{g} \mathrm{cm}^{-3}$ & $\phi_{\mathrm{v}} / \mathrm{cm}^{3} \cdot \mathrm{mol}^{-1}$ \\
\hline \multicolumn{4}{|c|}{$\phi_{1}=0.10$} \\
\hline \multirow{5}{*}{298.15} & 0.00168 & 0.98322 & 131.31 \\
\hline & 0.00224 & 0.98327 & 131.30 \\
\hline & 0.00325 & 0.98336 & 131.35 \\
\hline & 0.00684 & 0.98368 & 131.39 \\
\hline & 0.01122 & 0.98407 & 131.45 \\
\hline \multirow{5}{*}{308.15} & 0.00232 & 0.98116 & 134.72 \\
\hline & 0.00453 & 0.98135 & 134.83 \\
\hline & 0.00581 & 0.98146 & 134.87 \\
\hline & 0.00686 & 0.98155 & 134.92 \\
\hline & 0.01106 & 0.98191 & 135.03 \\
\hline \multirow{5}{*}{313.15} & 0.00176 & 0.97908 & 135.97 \\
\hline & 0.00341 & 0.97922 & 136.18 \\
\hline & 0.00518 & 0.97937 & 136.28 \\
\hline & 0.00719 & 0.97954 & 136.38 \\
\hline & 0.00944 & 0.97973 & 136.48 \\
\hline \multirow{5}{*}{318.15} & 0.00142 & 0.97637 & 137.11 \\
\hline & 0.00237 & 0.97645 & 137.21 \\
\hline & 0.00368 & 0.97656 & 137.37 \\
\hline & 0.00595 & 0.97675 & 137.58 \\
\hline & 0.01003 & 0.97709 & 137.87 \\
\hline \multicolumn{4}{|c|}{$\phi_{1}=0.20$} \\
\hline \multirow{4}{*}{298.15} & 0.00248 & 0.97069 & 129.43 \\
\hline & 0.00410 & 0.97084 & 129.50 \\
\hline & 0.00648 & 0.97106 & 129.59 \\
\hline & 0.00767 & 0.97117 & 129.61 \\
\hline \multicolumn{4}{|c|}{2041} \\
\hline
\end{tabular}


RASĀYAN J. Chem.

Vol. 12 | No. 4 |2039 - 2046| October - December | 2019

\begin{tabular}{|c|c|c|c|}
\hline & 0.01081 & 0.97146 & 129.67 \\
\hline \multirow{5}{*}{308.15} & 0.00155 & 0.96499 & 132.68 \\
\hline & 0.00366 & 0.96518 & 132.86 \\
\hline & 0.00611 & 0.96540 & 133.01 \\
\hline & 0.00834 & 0.96560 & 133.10 \\
\hline & 0.01035 & 0.96578 & 133.17 \\
\hline \multirow{5}{*}{313.15} & 0.00235 & 0.96221 & 134.09 \\
\hline & 0.00426 & 0.96238 & 134.25 \\
\hline & 0.00640 & 0.96257 & 134.40 \\
\hline & 0.00798 & 0.96271 & 134.49 \\
\hline & 0.01058 & 0.96294 & 134.62 \\
\hline \multirow{5}{*}{318.15} & 0.00214 & 0.95931 & 135.08 \\
\hline & 0.00520 & 0.95958 & 135.42 \\
\hline & 0.00645 & 0.95969 & 135.52 \\
\hline & 0.00850 & 0.95987 & 135.66 \\
\hline & 0.01041 & 0.96004 & 135.78 \\
\hline \multicolumn{4}{|c|}{$\phi_{1}=0.40$} \\
\hline \multirow{5}{*}{298.15} & 0.00150 & 0.93815 & 126.20 \\
\hline & 0.00441 & 0.93844 & 126.41 \\
\hline & 0.00662 & 0.93866 & 126.52 \\
\hline & 0.00773 & 0.93877 & 126.59 \\
\hline & 0.00914 & 0.93891 & 126.64 \\
\hline \multirow{5}{*}{308.15} & 0.00162 & 0.92378 & 129.45 \\
\hline & 0.00355 & 0.92397 & 129.66 \\
\hline & 0.00528 & 0.92414 & 129.78 \\
\hline & 0.00793 & 0.92440 & 129.91 \\
\hline & 0.00987 & 0.92459 & 130.00 \\
\hline \multirow{5}{*}{313.15} & 0.00131 & 0.91743 & 130.67 \\
\hline & 0.00438 & 0.91773 & 131.01 \\
\hline & 0.00663 & 0.91795 & 131.16 \\
\hline & 0.00786 & 0.91807 & 131.23 \\
\hline & 0.01022 & 0.91830 & 131.37 \\
\hline \multirow{5}{*}{318.15} & 0.00297 & 0.91571 & 131.87 \\
\hline & 0.00544 & 0.91595 & 132.10 \\
\hline & 0.00678 & 0.91608 & 132.18 \\
\hline & 0.00802 & 0.91620 & 132.28 \\
\hline & 0.00957 & 0.91635 & 132.37 \\
\hline
\end{tabular}

Representative plot (Fig.-1) records the changes in apparent molar volumes of the polyelectrolyte solutions as a function of the square root of the monomer concentration of NaCMC. Within the 
concentration range investigated here, the $\phi_{\mathrm{v}} \mathrm{Vs} \sqrt{c_{\mathrm{p}}}$ plots are found to be linear and, moreover, as $\sqrt{c_{\mathrm{p}}}$ is increased, $\phi_{\mathrm{v}}$ increased slightly. In the past, Conway and Desnoyers, ${ }^{22}$ Lawrence and Conway, ${ }^{23}$ Ise and $\mathrm{Okubo}^{24}$ and Tondre and Zana ${ }^{12}$ have reported similar linear increases for $\phi_{\mathrm{v}}$ concentration for various polyelectrolytes in aqueous solutions. However, the insensitivity of $\phi_{\mathrm{v}}$ toward concentration has also been reported ${ }^{24}$ for salts of polystyrene sulfonic acid polyethylenimine. It should be pointed out that the concentration dependences observed in the present study are small compared to those for most simple electrolytes. The positive slopes in acetonitrile-water mixed solvent media were probably due to the weakening of the ion-solvent interactions, which, in turn, was possibly due to appreciable counterion binding brought about by the increase in the polyelectrolyte concentration. This increase in the concentration of polyelectrolyte solutions must have led to the lowering of the contraction of the solvent medium resulting in a net positive volume change per monomole of the polyelectrolyte added, hence the positive slope of the $\phi_{\mathrm{v}}$ vs. $\sqrt{c_{\mathrm{p}}}$ plots.

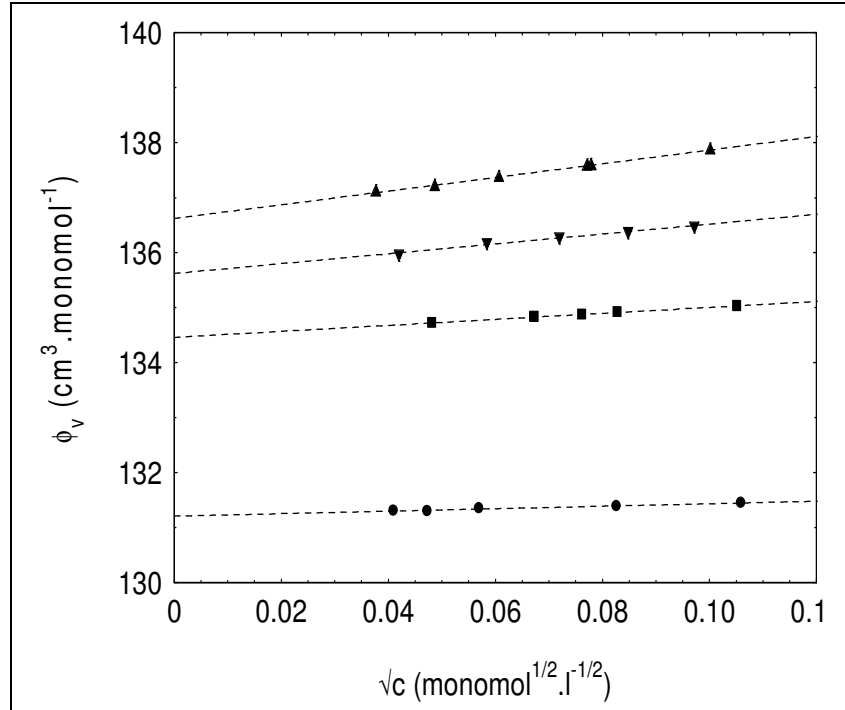

(a)

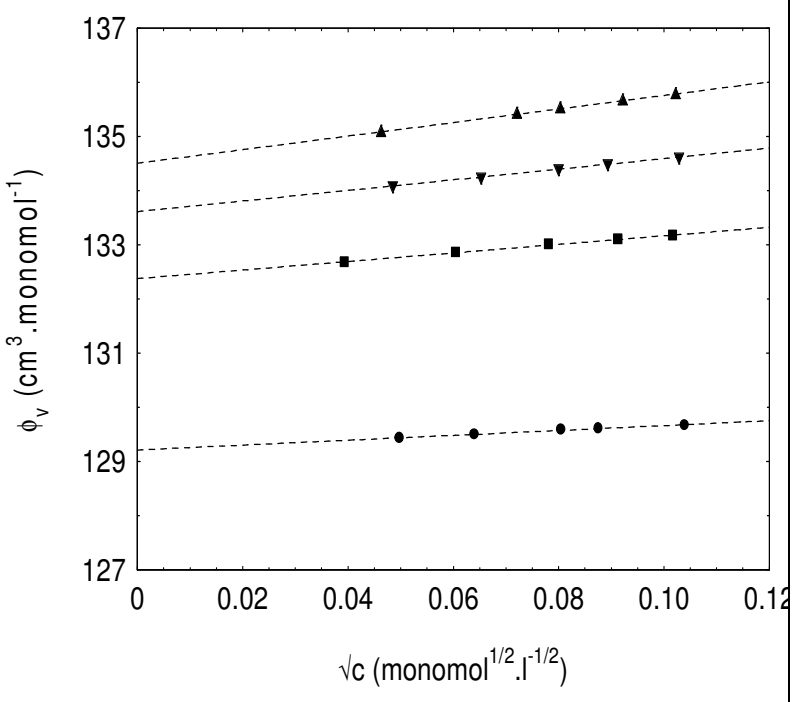

(b)

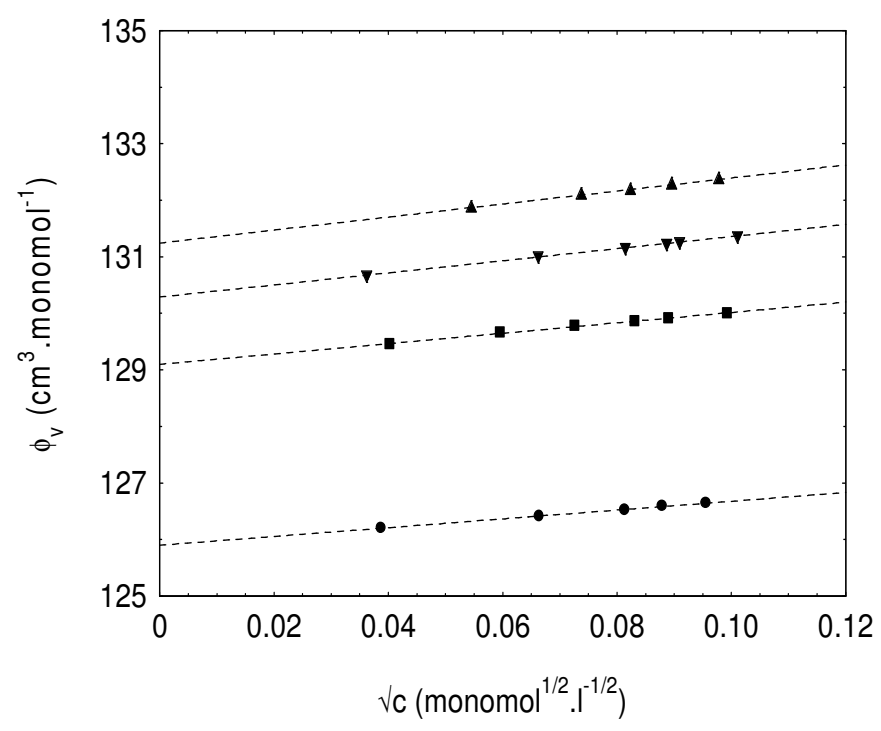

(c)

Fig.-1: Apparent Molar Volumes of NaCMC with (a) 0.10 (b) 0.20 and (c) 0.40 Volume Fraction of Acetonitrile in

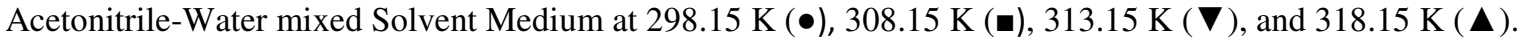


$\operatorname{Masson}^{25}$ equation was made use of to obtain the apparent molar volumes at infinite dilution, $\phi_{\mathrm{v}}^{0}\left(==_{V_{2}}^{0}\right)$ by the least-squares fitting of $\phi_{\mathrm{v}}$ values.

$$
\phi_{\mathrm{v}}=\phi_{\mathrm{v}}{ }^{0}+S_{\mathrm{v}} \sqrt{c_{\mathrm{p}}}
$$

Where $S_{\mathrm{v}}$ is the experimental slope. Table-3 shows the apparent molar volumes at infinite dilution, $\phi_{\mathrm{v}}^{0}$ $\left(=\bar{V}_{2}^{0}\right)$ at different temperatures. Regression analysis of the data gives a correlation coefficient $(r)$ of $\geq$ 0.985. At a given temperature, when the medium is enriched with acetonitrile there is a decrease in the limiting partial molar volumes. But, as the temperature increases in a given mixed solvent $V_{2}^{-0}$ increases.

Table-3: Limiting Partial Molar Volumes, $\stackrel{-}{V}_{2} / \mathrm{Cm}^{3} \cdot \mathrm{Mol}^{-1}$ of NaCMC in Acetonitrile-Water Mixed Solved Media At $(298.15,308.15,313.15$, And 318.15) K

\begin{tabular}{c|c|c|c}
\hline \multirow{2}{*}{$T / \mathrm{K}$} & \multicolumn{3}{|c}{$\bar{V}_{2}^{0} / \mathrm{cm}^{3} \cdot \mathrm{mol}^{-1}$} \\
\cline { 2 - 4 } & $\phi_{1}=0.10$ & $\phi_{1}=0.20$ & $\phi_{1}=0.40$ \\
\hline 298.15 & $131.21 \pm 0.02$ & $129.21 \pm 0.02$ & $125.90 \pm 0.01$ \\
308.15 & $134.46 \pm 0.01$ & $132.38 \pm 0.02$ & $129.10 \pm 0.02$ \\
313.15 & $135.62 \pm 0.05$ & $133.62 \pm 0.01$ & $130.29 \pm 0.01$ \\
318.15 & $136.62 \pm 0.02$ & $134.51 \pm 0.02$ & $131.24 \pm 0.02$ \\
\hline
\end{tabular}

To investigate the specific behavior of the polyion and the counterion comprising the polyelectrolyte it is necessary to split the $\bar{V}_{2}^{0}$ values into their ionic components. Now, the limiting partial molar volumes of sodium ion could be obtained from the literature values of $\bar{V}_{2}^{0}$ of $\mathrm{NaCl}$, sodium tetraphenylborate $\left(\mathrm{NaBPh}_{4}\right)$, and tetraphenylphosphoniun chloride $\left(\mathrm{Ph}_{4} \mathrm{PCl}\right)$ in acetonitrile-water mixtures at $298.15 \mathrm{~K}^{26}$ by employing the tetraphenylphosphoniun tetraphenylborate (TPTB) assumption. ${ }^{27} \mathrm{We}$, therefore, have separated the limiting partial molar volumes of $\mathrm{NaCMC}$ in acetonitrile-water mixtures in the given condition. The limiting partial molar volumes of sodium ion are, however, not available at the relevant experimental compositions of the acetonitrile-water mixtures. The already known values of the limiting partial molar volumes of $\mathrm{NaCl}, \mathrm{NaBPh}_{4}$, and $\mathrm{Ph}_{4} \mathrm{PCl}$ in acetonitrile-water mixtures at $298.15 \mathrm{~K}$ were plotted vis-à-vis the volume fraction of acetonitrile in acetonitrile-water mixtures. Then the $\bar{V}_{2}^{0}$ values of these electrolytes were extrapolated in the context of the present investigation. The result was then used to get the limiting partial molar volume of sodium ion via the TPTB assumption.

In Millero's, ${ }^{28,29}$ work the ionic limiting partial molar volume, $V_{i o n}^{-0}$, was considered as the combination of the following components:

$$
V_{\text {ion }}^{-^{0}}=V_{\text {int } r}^{-^{0}}+V_{\text {elec }}^{-^{0}}+\bar{V}_{\text {str }}^{-^{0}}+V_{\text {cage }}^{-^{0}}
$$

Where, $V_{\text {int } r}^{-0}$, the intrinsic partial molar volume, is the minute positive change in the $V_{\text {ion }}^{-0}$ caused by the intrinsic volume of the ion is as per the rule intrinsic ionic volume in a solution is equal to its crystallographic volume. ${ }^{30} V_{\text {elec }}^{-0^{0}}$, the electrostriction partial molar volume, is the minute negative change which is equal to the decrease in molar solute volume caused by ion-solvent electrostrictive interaction. $V_{s t r}^{-0}$ is the void-space partial molar volume which is illumined by minute change caused by the destruction of solvent structure in the region of the ionic co-sphere. $V_{\text {cage }}^{-0}$ is the caged partial molar volume. This refers to the minute negative change which is characteristic of solvophobic "structuremaking" parts of the ions in solution. This, in turn, is caused by the filling of the intermolecular cavities of the solvent structure. It may be pointed out that the electric forces due to the negative charges on the structural units of $\mathrm{NaCMC}$ affect strongly the surrounding solvent molecules and hence for the 
carboxymethylcellulose polyion, $V_{\text {elec }}^{-^{0}}$ will contribute significantly to its limiting partial molar volume. Because of the polar nature of acetonitrile-water mixtures, the apolar part will also contribute to the polyion limiting partial molar volume through the term $V_{\text {cage }}^{-{ }^{0}}$. Sodium-ion, on the other hand, has nothing to do with caged partial molar volume.

Table-4 indicates that as the acetonitrile-water mixtures are enriched with acetonitrile, the limiting partial molar volume of the polyion decreases. This indicates that the total contribution of the terms $V_{\text {elec }}^{-0}$ and $V_{\text {cage }}^{-{ }^{0}}$ becomes more important over that of the term $V_{s t r}^{-0}$ as the solvent medium is enriched in acetonitrile. This is a manifestation of the predominance of the combined influence of the polyion-solvent electrostrictive interaction and the solvophobic filling of the intermolecular cavities of the solvent structure by the apolar parts of the polyion with increasing acetonitrile content in the medium. For sodium ion, on the other hand, the disordered partial molar volume plays a leading role. In the case of the polyelectrolyte as a whole, however, the polyion is governing the solution behavior. Here also the limiting partial molar volume of NaCMC decreases with augmentation of acetonitrile in the acetonitrile-water mixtures (Table-3) like the polyion. An increase in the temperature increases the limiting partial molar volume of NaCMC in a given mixed solvent medium as can be seen from Table-3. This might be ascribed to the growing importance of the disordered partial molar volume owing to a temperature-induced desolvation of the counterion leading to more counterion binding at higher temperatures as observed earlier from conductivity measurements. ${ }^{31}$

Table-4: Limiting Partial Molar Volumes, $\bar{V}_{2}^{0}\left(\mathrm{Cm}^{3} . \mathrm{Mol}^{-1}\right)$ of Sodium Ion $\left(\mathrm{Na}^{+}\right)$And Carboxymethylcellulose Polyion ( $\left.\mathrm{CMC}^{-}\right)$In Acetonitrile-Water Mixed Solvent Media At 298.15 K

\begin{tabular}{c|c|c|c}
\hline \multirow{3}{*}{ Ion / Polyion } & \multicolumn{3}{|c}{$\bar{V}_{2}^{-0} / \mathrm{cm}^{3} \cdot \mathrm{mol}^{-1}$} \\
\cline { 2 - 4 } & $\phi_{1}=0.10$ & $\phi_{1}=0.20$ & $\phi_{1}=0.40$ \\
\hline $\mathrm{Na}^{+}$ & -9.12 & -7.14 & -3.35 \\
$\mathrm{CMC}^{-}$ & 140.33 & 136.35 & 129.25 \\
\hline
\end{tabular}

\section{CONCLUSION}

The present investigation indicates that in acetonitrile-water mixed solvent media as we increase the concentration of the polyelectrolyte, the counterion binding increases noticeably and thereby weakens the ion-solvent interactions. Moreover, the polyion is found to govern the volumetric behavior of the polyelectrolyte as a whole in these solutions. Besides, there is a predominant influence of both the polyion-solvent electrostrictive interactions and the solvophobic filling of the intermolecular cavities of the solvent structure which are seen to be caused by the apolar parts of the polyion when the medium was enriched by acetonitrile. A temperature-induced desolvation of the counterions leading to more counterion binding at higher temperatures was also inferred from this study.

\section{ACKNOWLEDGMENT}

We are grateful to Professor Bijan Das, Department of Chemistry, Presidency University, 86/1 College Street, Kolkata 700 073, India for providing valuable inputs. We also thank the Head, Department of Chemistry, University of North Bengal, Darjeeling and Principal, NBBD College, Tadong for extending their institutional facilities in the carrying out of this work. The authors sincerely acknowledge the help extended by Dr. Mukund Giri, Associate professor, NBBD College, Tadong in manuscript writing.

\section{REFERENCES}

1. T. Yamase and M.T. Pope, Polyoxometalate Chemistry for Nano-Composite Design, Kluwer Academic/Plenum Publishers, New York, (2002).

2. M.T. Pope and A. Muller, Polyoxometalate Chemistry: From Topology via Self- Assembly to Applications, Kluwer Academic Publishers, Netherland, (2001). 
3. M.A.C. Stuart, W.T.S. Huck, J. Genzer, M. Muller, M. Ober, C. Stamm, G.B. Sukhorukov, I. Szleifer, V.V. Tsukruk, M. Urban, F. Winnik, S. Zauscher, I. Luzinov and S. Minko, Nat. Materials, 9, 101 (2010), DOI: 10.1038/nmat2614

4. J.H. Ortony, T. Chatterjee, L.E. Garner, A. Chworoz, A. Mikhailowsky, E.J. Kramer and G.C. Bazan, J. Am. Chem. Soc., 133, 8380 (2011), DOI: 10.1021/ja202776b

5. C.K. Materese, A. Savelyev and G.A. Papoian, J. Am. Chem. Soc., 131, 15005 (2009), DOI: 10.1021/ja905376q

6. A. Chatterjee, B. Das and C. Das, Carbohydrate Polym., 87, 1144 (2012), DOI: 10.1016/j.carbpol.2011.08.087

7. R. Sharma, C. Das, S. Dahal and B. Das, Carbohydrate Polym., 92, 1546 (2013), DOI: 10.1016/j.carbpol.2012.11.012

8. C. Das and B. Das, J. Chem. Therms., 68, 293 (2014), DOI: 10.1016/j.jct.2013.09.022

9. C. Das, B. Sharma and B. Das, J. Mol. Liq. 2016; 219: 104 (2016), DOI: 10.1016/j.molliq.2016.03.030

10. A. Ikegami, Biopolym., 6, 431 (1968), DOI: 10.1002/bip.1968.360060314

11. J. Skerjanc, J. Phys. Chem., 77, 2225 (1973), DOI: 10.1021/j100637a013

12. C. Tondre and R. Zana, J. Phys. Chem., 76, 3451(1972), DOI: 10.1021/j100667a026

13. F. Shahidi, J. Soln. Chem., 12, 295 (1983), DOI: 10.1007/BF00646204

14. R. C. Thakur, R. Sharma and V. Gill, Rasayan J Chem., 9(1), 44 (2016).

15. M. Luksic and B.H. Lee, J. Mol. Liq., 228, 126 (2017), DOI:10.1016/j.molliq.2016.09.043

16. R. Sadeghi, R. Khoshnavazi, H. Parhizgar and L. Bahrami, Fluid Phase Eqa., 277, 87 (2009), DOI:10.1016/j.fluid.2008.11.009

17. T. Sumathi and M. Varalakshmi, Rasayan J. Chem., 3(3), 550 (2010).

18. G. Moumouzias,D. K. Panopoulo and G.J. Ritzoulis, J. Chem. Eng. Data,36, 20 (1991), DOI: 10.1021/je00001a006

19. R.A. Robinson and R.H. Stokes, Electrolyte Solutions, Butterworths, London, (1959).

20. M. Pal and S. Bagchi, J. Chem. Soc. Faraday Trans. 1, 81, 961 (1985), DOI:10.1039/F19858100961

21. A. Domard and M. Rinaudo, Int. J. Biol. Macromol., 5, 49 (1983), DOI:10.1016/01418130(83)90078-8

22. B. Conway, J.E. Desnoyers and A.C. Smith, Phil. Trans. Royal Soc. A, 256, 359 (1964), DOI: 10.1098/rsta.1964.0010

23. B. Conway and J. Lawrence, J. Phys. Chem., 75, 2353 (1971), DOI: 10.1021/j100684a021

24. N. Ise and T. Okubo, J. Am.Chem. Soc., 90, 4527 (1968), DOI: 10.1021/ja01019a003

25. D.O. Masson, Philos. Mag., 8, 218 (1929), DOI:10.1080/14786440808564880

26. G.T. Hefter, J.P.E. Grolier and A.H. Roux, G. Roux-Desgranges, J. Soln. Chem., 19, 207 (1990), DOI: $10.1007 / \mathrm{BF} 00650455$

27. M.H. Abraham and Y. Marcus, J. Chem. Soc., Faraday Trans. 1, 82, 3255 (1986), DOI: 10.1039/F19868203255

28. F.J. Millero,1971,In Biophysical Properties of the Skin, in: H. R. Elden (Eds.), John Wiley, New York

29. F.J. Millero, 1972, In Structure and Transport Processes in Water and Aqueous Solutions, in: R. A. Horne (Eds.), John Wiley, New York

30. F.J. Millero, J. Phys. Chem., 73, 2417 (1969), DOI: 10.1021/j100727a056

31. P. Nandi and B. Das, J. Phys. Chem. B, 109, 3238 (2005), DOI: 10.1021/jp045801t

[RJC-5388/2019] 